\title{
Who we are: Demographic and Stress Profile of the Philippine LIFECARE Cohort
}

\author{
Olivia T. Sison, ${ }^{1,2,3}$ Queenie G. Ngalob, ${ }^{3}$ Felix Eduardo R. Punzalan, $, 3,4$ Nina T. Castillo-Carandang, ${ }^{1,3}$ \\ Paulette D. Nacpil-Dominguez, ${ }^{3}$ Elmer Jasper B. Llanes, ${ }^{3,4}$ Felicidad V. Velandria, ${ }^{3}$ Paul Ferdinand M. Reganit, ${ }^{3,4}$ \\ Wilbert Allan G. Gumatay ${ }^{3}$ and Rody G. Sy $y^{3,4,5}$ on behalf of the LIFECARE Philippines Team \\ ${ }^{1}$ Department of Clinical Epidemiology, College of Medicine, University of the Philippines Manila \\ ${ }^{2}$ Institute of Clinical Epidemiology, National Institutes of Health, University of the Philippines Manila \\ ${ }^{2}$ LIFE course study in CARdiovascular disease Epidemiology (LIFECARE) Philippine Study Group, Lipid Research Unit, UP-PGH, Manila, Philippines \\ ${ }^{3}$ Department of Medicine, College of Medicine and Philippine General Hospital University of the Philippines Manila \\ ${ }^{4}$ Cardinal Santos Medical Center, San Juan City, Metro Manila, Philippines
}

\begin{abstract}
Objective. To describe the demographic and stress profile of the participants in the LIFECARE cohort.

Methods. The Life Course Study in Cardiovascular Disease Epidemiology (LIFECARE) is a community-based prospective cohort of apparently healthy individuals aged 20 to 50 years old with no preexisting cardiovascular disease. The second phase out of four phases of the study involves collection of baseline socio-demographic, anthropometric, biochemical and cardiovascular parameters and stress profiles. It was conducted via face-to-face interview using a survey questionnaire.

Results and Conclusion. A total of 3072 participants from Metro Manila and 4 provinces in Luzon were recruited. The female to male ratio was 1.3:1. Majority of the participants were aged 30 years old and above. Most were married, employed and literate. Majority attained at least high school level of education. Loss of job was the most common stressor experienced in the past year. Majority of the cohort reported occasional experience of general stress and moderate level of financial stress. Occurrence of general stress within the past year was higher among females ( $p$ $<0.001)$, younger age-group $(p=0.006)$, and among those who reached college level of education $(p<0.001)$. Furthermore, level of current financial stress was high to severe among older agegroup $(p=0.004)$, and among widow/widower/separated $(p<0.0001)$. While the relationship between psychosocial stress and physical illness had not been established in this study, there is a need to investigate demographics and psychosocial stress, and their implications in increasing adverse health outcomes in general, and cardiovascular risk in particular.
\end{abstract}

Key Words: LIFECARE, cardiovascular risk factors, Philippines, demographic profile, stress

Corresponding author: Olivia T. Sison, MSc

Department of Clinical Epidemiology

College of Medicine

University of the Philippines Manila

Rm.103 - Paz Mendoza Bldg.

547 Pedro Gil Street, Ermita, Manila 1000 Philippines

Telephone: +6325228380

Fax No.: +632 5254098

Email: olivia_sison@yahoo.com

\section{Introduction}

Both in the local and global settings, diseases of the heart and vascular system are leading health care issues. Since 1975, a steady rise in the prevalence of heart diseases was seen among Filipinos. ${ }^{1}$ Diseases of the heart and vascular system remained the top two causes of mortality from 1988 until the present. ${ }^{2}$ Because of the impact of cardiovascular diseases (CVD), it has become a universal goal to control traditional risk factors that contribute to its occurrence.

In recent years, there is growing interest on the effect of socioeconomic factors and psychosocial stress in the development and progression of CVD. Cross-sectional studies reveal an inverse relationship between prevalence of cardiovascular (CV) risk factors and socioeconomic factors such as occupation, level of education and individual family income. ${ }^{3-6}$ Marital status was also reported to affect occurrence of CVD and its risk factors. Among Thai women, being married or having a partner were reported to be associated with hypertension. ${ }^{7}$ This was in contrast to African-Americans where being single conferred an increased likelihood of death for men. ${ }^{8}$ A large body of literature demonstrates invariable effects of psychosocial stress on cardiovascular risk. Data from the White Hall II Study which observed 7268 adults over a maximum of 18.3 years showed that participants who perceived stress to impact their health "a lot or extremely" had a 2.12 fold higher risk of coronary deaths or incident nonfatal myocardial infarction. These individuals were also shown to have a survival disadvantage compared to those who perceived that stress did not affect their health. ${ }^{9}$ Data on the epidemiology of socio-demographic risk factors and stress in the Asian and Philippine population have yet to be determined.

The LIFE course study in CARdiovascular disease Epidemiology (LIFECARE) was conceptualized to determine the effect of socioeconomic and lifestyle factors and psychosocial stress in the development of cardiovascular disease (CVD). It will also attempt to determine the effect of 
CVD on health care utilization and quality of life. It is a collaboration between four Southeast Asian countries namely Philippines, Malaysia, Indonesia and Thailand in central coordination with Singapore. It is a cross-sectional survey conducted in 2009 with a planned repeat visit in 2013-2014. This paper will describe the baseline demographic characteristics of the Philippine cohort derived from the initial survey conducted in 2009.

\section{Objective}

To describe the demographic and stress profile of the participants of the Philippine LIFECARE cohort.

\section{Materials and Methods}

The Life Course Study in Cardiovascular Disease Epidemiology (LIFECARE) is a community-based prospective cohort of apparently healthy individuals aged 20 to 50 years old conducted last 2009 to 2011. Excluded were those who have existing CVD as determined by the respondent's medical history (previous myocardial infarction $[\mathrm{MI}]$, stroke, peripheral arterial disease (PAD); history of malignancies (treated or otherwise); had plans to migrate outside their community within the next 5 years; pregnant, breastfeeding or lactating women and those who are eligible but at home only once a month which would make them unavailable for follow-up.

Provinces from Luzon were selected by convenience sampling and included Metro Manila, Rizal, Batangas, Bulacan and Quezon. A total of 62 barangays (villages) were selected based on the relatively good accessibility, peace and order situation, presence of support from the local government unit (LGU) and local collaborators. LGU officials facilitated the identification of barangays for inclusion in the study. Household lists from each barangay were obtained from the LGUs and random selection of households was done using systematic sampling. A total of 120 households were randomly selected from each barangay. Only one eligible participant per household was chosen using the Kish method in order to have a balanced distribution of age and sex of study participants from the community.

Socio-demographic profile, medical history and physical examination were conducted on all participants. Anthropometric data were obtained and blood samples were taken for baseline metabolic profile. Electrocardiogram (ECG) and ankle brachial indices were also tested for all participants.

Perceived stress was determined via intervieweradministered questionnaire. Stress was defined as feeling irritable, anxious or having sleeping difficulties as a result of conditions at work or at home. To assess occurrence of stressors, participants were asked the question "Nakaranas na po ba kayo ng alinman sa mga sumusunod noong nakaraang taon?" ("Have you experienced any of the following in the past year?"). Responses were categorized into 10 stressors as listed in Table 5. To assess the frequency of perceived stress, the participants were asked the following question: "Gaano po kadalas kayo nakaramdam ng stress nitong nakaraang taon?" ("How often have you felt stress at work in the past year?"). Responses were categorized in a 4-point scale: never, some periods, several periods or permanent stress. To assess the level of perceived financial stress, the following question was asked: "Ano po ba ang antas ng stress na pangpinansyal/ pampera ang inyong nararanasan?" ("What level of financial stress do you feel?"). Responses were categorized in a 4point scale: none, little, moderate and high/severe financial stress.

A detailed methodology was described in previous publications of the Philippine LIFECARE Study. ${ }^{10,11}$

\section{Results}

A total of 3072 patients were included in the Philippine cohort of the LIFECARE Study. The patients were recruited from 4 provinces (Bulacan, Batangas, Quezon and Rizal) and three cities from Metro Manila (Makati, Manila and Marikina) in Luzon. In total, the participants were sampled from 62 barangays (villages) of 10 cities/municipalities (towns). Bulacan province had the highest number of participants recruited while Metro Manila had the least. There were more females in all areas recruited at a cumulative female to male ratio of 1.3:1 (Table 1). Majority of the participants are 30 years old and above (Table 2).

Table 1. Frequency and percent distribution of participants by sex for each study site

\begin{tabular}{lccc}
\hline & $\begin{array}{c}\text { Male } \\
\text { No. }(\mathbf{\%})\end{array}$ & $\begin{array}{c}\text { Female } \\
\text { No. } \mathbf{( \% )}\end{array}$ & Total \\
\hline Metro Manila & 59 & 125 & 184 \\
(8 barangays) & $(32.1)$ & $(67.9)$ & \\
Bulacan & 414 & 555 & 969 \\
(16 barangays) & $(42.7)$ & $(57.3)$ & \\
Quezon & 364 & 445 & 809 \\
(16 barangays) & $(45.0)$ & $(55.0)$ & \\
Batangas & 362 & 454 & 816 \\
(16 barangays) & $(44.4)$ & $(55.6)$ & \\
Rizal & 130 & 164 & 294 \\
(6 barangays) & $(44.2)$ & $(55.8)$ & 3072 \\
Total & 1329 & 1743 & \\
& $(43.3)$ & $(56.7)$ & \\
\hline
\end{tabular}

\section{Civil Status}

Majority of the respondents were married. A significant proportion was single and they were mostly males. A small proportion of the cohort was separated and/or widowed (Table 2).

\section{Employment}

Two-thirds of the participants were currently employed at the time of the survey. While more females were included in the cohort, more males participated in the workforce. A third of those in the workforce was either 
regularly or self-employed. This proportion was similarly reflected in the individual areas of Metro Manila, Quezon and Batangas. Regular employment was highest in Bulacan and Rizal provinces (Table 3). Across all areas sampled, unemployment rate was approximately one-third of the study population, with the highest proportion in Metro Manila.

Table 2. Frequency and percent distribution of participants by demographic characteristics

\begin{tabular}{lccc}
\hline & $\begin{array}{c}\text { Male } \\
\mathbf{n}=\mathbf{1 3 2 9}\end{array}$ & $\begin{array}{c}\text { Female } \\
\mathbf{n}=\mathbf{1 7 4 3}\end{array}$ & $\begin{array}{c}\text { TOTAL } \\
\mathbf{n}=\mathbf{3 0 7 2}\end{array}$ \\
\hline Age & & & \\
$20-29$ & $402(30.2)$ & $459(26.3)$ & $861(28.0)$ \\
$30-39$ & $477(35.9)$ & $625(35.9)$ & $1,102(35.9)$ \\
$40-50$ & $450(33.9)$ & $659(37.8)$ & $1,109(36.1)$ \\
Civil Status & & & \\
Single & $334(25.1)$ & $247(14.2)$ & $581(18.9)$ \\
Married & $801(60.3)$ & $1217(69.8)$ & $2018(65.7)$ \\
Widow/ Widower & $10(0.8)$ & $55(3.2)$ & $65(2.1)$ \\
Separated & $24(1.8)$ & $32(1.8)$ & $56(1.8)$ \\
Live-in & $160(12.0)$ & $192(11.0)$ & $352(11.5)$ \\
Employment Status & & & \\
Employed (regular) & $431(32.4)$ & $313(18.0)$ & $744(24.2)$ \\
Employed & $270(20.3)$ & $131(7.5)$ & $401(13.1)$ \\
(not regular) & & & \\
Self-employed & $444(33.4)$ & $480(27.8)$ & $924(30.1)$ \\
Retired/Student & $17(1.3)$ & $23(1.3)$ & $40(1.3)$ \\
Unemployed & $167(12.6)$ & $796(45.7)$ & $963(31.4)$ \\
Educational Attainment & & & \\
No formal schooling & $4(0.3)$ & $3(0.2)$ & $7(0.2)$ \\
Elementary & $303(22.8)$ & $312(17.9)$ & $615(20.0)$ \\
High School & $605(45.5)$ & $797(45.7)$ & $1402(45.6)$ \\
Vocational & $133(10.0)$ & $115(6.6)$ & $248(8.1)$ \\
College & $280(21.1)$ & $514(29.5)$ & $794(25.9)$ \\
Post-graduate & $4(0.3)$ & $2(0.1)$ & $6(0.2)$ \\
\hline & & &
\end{tabular}

Table 3. Frequency and percent distribution of participants by employment status for each study site

\begin{tabular}{lcccccc}
\hline $\begin{array}{c}\text { Employment } \\
\text { Status }\end{array}$ & $\begin{array}{c}\text { Metro } \\
\text { Manila } \\
(\mathbf{n = 1 8 4 )}\end{array}$ & $\begin{array}{c}\text { Bulacan } \\
(\mathbf{n}=\mathbf{9 6 9 )}\end{array}$ & $\begin{array}{c}\text { Quezon } \\
\mathbf{( n = 8 0 9 )}\end{array}$ & $\begin{array}{c}\text { Batangas } \\
(\mathbf{n}=\mathbf{8 1 6})\end{array}$ & $\begin{array}{c}\text { Rizal } \\
(\mathbf{n}=\mathbf{2 9 4})\end{array}$ & $\begin{array}{c}\text { TOTAL } \\
(\mathbf{n}=\mathbf{3 0 7 2})\end{array}$ \\
\hline Employed & 38 & 292 & 156 & 174 & 84 & 744 \\
(regular) & $(20.65)$ & $(30.13)$ & $(19.28)$ & $(21.32)$ & $(28.57)$ & $(24.22)$ \\
Employed & 21 & 123 & 105 & 107 & 45 & 401 \\
(not regular) & $(11.41)$ & $(12.69)$ & $(12.98)$ & $(13.11)$ & $(15.31)$ & $(13.05)$ \\
Self- & 42 & 252 & 289 & 275 & 66 & 924 \\
employed & $(22.83)$ & $(26.01)$ & $(35.72)$ & $(33.70)$ & $(22.45)$ & $(30.08)$ \\
Retired / & 5 & 11 & 8 & 9 & 7 & 40 \\
Student & $(2.72)$ & $(1.14)$ & $(0.99)$ & $(1.10)$ & $(2.38)$ & $(1.30)$ \\
Unemployed & 78 & 291 & 251 & 251 & 92 & 963 \\
& $(42.39)$ & $(30.03)$ & $(31.03)$ & $(30.76)$ & $(31.29)$ & $(31.35)$ \\
\hline
\end{tabular}

\section{Educational Attainment}

The vast majority of the cohort had formal education (99.8\%). Majority achieved at least high school level (Table 4). Metro Manila and Rizal had the highest proportion of participants who achieved college education. High school level was the highest attainment for most of the respondents from Bulacan, Quezon and Batangas (Table 4).
Table 4. Frequency and percent distribution of participants by educational attainment for each study site

\begin{tabular}{lcccccc}
\hline $\begin{array}{l}\text { Educational } \\
\text { Attainment }\end{array}$ & $\begin{array}{c}\text { Metro } \\
\text { Manila } \\
(\mathbf{n}=\mathbf{1 8 4})\end{array}$ & $\begin{array}{c}\text { Bulacan } \\
(\mathbf{n}=\mathbf{9 6 9 )}\end{array}$ & $\begin{array}{c}\text { Quezon } \\
(\mathbf{n}=\mathbf{8 0 9})\end{array}$ & $\begin{array}{c}\text { Batangas } \\
(\mathbf{n}=\mathbf{8 1 6})\end{array}$ & $\begin{array}{c}\text { Rizal } \\
(\mathbf{n}=\mathbf{2 9 4})\end{array}$ & $\begin{array}{c}\text { TOTAL } \\
(\mathbf{n}=\mathbf{3 0 7 2})\end{array}$ \\
\hline No formal & 0 & 1 & 5 & 1 & 0 & 7 \\
education & & $(0.1)$ & $(0.6)$ & $(0.1)$ & & $(0.2)$ \\
Elementary & 13 & 225 & 243 & 117 & 17 & 615 \\
& $(7.1)$ & $(23.2)$ & $(30.0)$ & $(14.3)$ & $(5.8)$ & $(20.0)$ \\
High & 71 & 420 & 366 & 429 & 116 & 1402 \\
School & $(38.6)$ & $(43.3)$ & $(45.2)$ & $(52.6)$ & $(39.5)$ & $(45.6)$ \\
Vocational & 17 & 73 & 61 & 68 & 29 & 248 \\
& $(9.2)$ & $(7.5)$ & $(7.5)$ & $(8.3)$ & $(9.9)$ & $(8.1)$ \\
College & 83 & 248 & 134 & 199 & 130 & 794 \\
& $(45.1)$ & $(25.6)$ & $(16.6)$ & $(24.4)$ & $(44.2)$ & $(25.9)$ \\
Post- & 0 & 2 & 0 & 2 & 2 & 6 \\
graduate & & $(0.2)$ & & $(0.2)$ & $(0.7)$ & $(0.2)$ \\
\hline
\end{tabular}

Demographics and stress

Employment-related issues comprised the most common stressors experienced by the cohort. Loss of job was the most prevalent across all study areas with the highest proportion seen in Metro Manila and Batangas. Loss of crop or failure in business was the second most common stressor. Health-related events contributed to a significant proportion of stressors second to employment. This included major illness in the family and serious personal injury or death. Familial concerns such as death of spouse or family members, intra-family conflicts and marital separation had the lowest contribution to the sources of stress (Table 5).

Table 5. Frequency and percent distribution of participants by area who experienced stress within the past year

\begin{tabular}{|c|c|c|c|c|c|c|}
\hline $\begin{array}{c}\text { Events } \\
\text { experienced }\end{array}$ & $\begin{array}{l}\text { Metro } \\
\text { Manila } \\
(\mathrm{n}=184)\end{array}$ & $\begin{array}{c}\begin{array}{c}\text { Bulacan } \\
(n=969)\end{array}\end{array}$ & $\begin{array}{r}\text { Quezon } \\
(n=809)\end{array}$ & $\begin{array}{c}\text { Batangas } \\
(n=816)\end{array}$ & $\begin{array}{c}\text { Rizal } \\
(n=294)\end{array}$ & $\begin{array}{l}\text { TOTAL } \\
(\mathrm{n}=3072)\end{array}$ \\
\hline Loss of Job & $\begin{array}{c}64 \\
(34.8)\end{array}$ & $\begin{array}{c}291 \\
(30.0)\end{array}$ & $\begin{array}{c}274 \\
(33.9)\end{array}$ & $\begin{array}{c}283 \\
(34.7)\end{array}$ & $\begin{array}{c}78 \\
(26.5)\end{array}$ & $\begin{array}{c}990 \\
(32.2)\end{array}$ \\
\hline Retirement & $\begin{array}{c}6 \\
(3.3)\end{array}$ & $\begin{array}{c}21 \\
(2.2)\end{array}$ & $\begin{array}{c}21 \\
(2.6)\end{array}$ & $\begin{array}{c}20 \\
(2.4)\end{array}$ & $\begin{array}{c}10 \\
(3.4)\end{array}$ & $\begin{array}{c}78 \\
(2.5)\end{array}$ \\
\hline $\begin{array}{l}\text { Loss of crop/ } \\
\text { business failure }\end{array}$ & $\begin{array}{c}25 \\
(13.6)\end{array}$ & $\begin{array}{c}109 \\
(11.2)\end{array}$ & $\begin{array}{c}187 \\
(23.1)\end{array}$ & $\begin{array}{c}215 \\
(26.4)\end{array}$ & $\begin{array}{c}28 \\
(9.5)\end{array}$ & $\begin{array}{c}564 \\
(18.4)\end{array}$ \\
\hline Violence & $\begin{array}{c}3 \\
(1.6)\end{array}$ & $\begin{array}{c}33 \\
(3.4)\end{array}$ & $\begin{array}{c}25 \\
(3.1)\end{array}$ & $\begin{array}{c}22 \\
(2.7)\end{array}$ & $\begin{array}{c}20 \\
(6.8)\end{array}$ & $\begin{array}{l}103 \\
(3.4)\end{array}$ \\
\hline $\begin{array}{l}\text { Serious personal } \\
\text { injury or illness }\end{array}$ & $\begin{array}{c}12 \\
(6.5)\end{array}$ & $\begin{array}{c}63 \\
(6.5)\end{array}$ & $\begin{array}{c}51 \\
(6.3)\end{array}$ & $\begin{array}{c}51 \\
(6.2)\end{array}$ & $\begin{array}{c}18 \\
(6.1)\end{array}$ & $\begin{array}{l}195 \\
(6.4)\end{array}$ \\
\hline $\begin{array}{l}\text { Marital } \\
\text { separation/ } \\
\text { annulment/ } \\
\text { divorce }\end{array}$ & $\begin{array}{c}5 \\
(2.7)\end{array}$ & $\begin{array}{c}26 \\
(2.7)\end{array}$ & $\begin{array}{c}11 \\
(1.4)\end{array}$ & $\begin{array}{c}11 \\
(1.4)\end{array}$ & $\begin{array}{c}10 \\
(3.4)\end{array}$ & $\begin{array}{c}63 \\
(2.1)\end{array}$ \\
\hline Death of spouse & $\begin{array}{c}2 \\
(1.1)\end{array}$ & $\begin{array}{c}8 \\
(0.8)\end{array}$ & $\begin{array}{c}8 \\
(1.0)\end{array}$ & $\begin{array}{c}4 \\
(0.5)\end{array}$ & $\begin{array}{c}1 \\
(0.3)\end{array}$ & $\begin{array}{c}23 \\
(0.8)\end{array}$ \\
\hline $\begin{array}{l}\text { Death of close } \\
\text { family member }\end{array}$ & $\begin{array}{c}27 \\
(14.7)\end{array}$ & $\begin{array}{c}59 \\
(6.1)\end{array}$ & $\begin{array}{c}39 \\
(4.8)\end{array}$ & $\begin{array}{c}47 \\
(5.8)\end{array}$ & $\begin{array}{c}15 \\
(5.1)\end{array}$ & $\begin{array}{l}187 \\
(6.1)\end{array}$ \\
\hline $\begin{array}{l}\text { Major illness of } \\
\text { a close family } \\
\text { member }\end{array}$ & $\begin{array}{c}26 \\
(14.1)\end{array}$ & $\begin{array}{c}126 \\
(13.0)\end{array}$ & $\begin{array}{c}92 \\
(11.4)\end{array}$ & $\begin{array}{c}101 \\
(12.4)\end{array}$ & $\begin{array}{c}53 \\
(18.0)\end{array}$ & $\begin{array}{c}398 \\
(13.0)\end{array}$ \\
\hline $\begin{array}{l}\text { Major intra- } \\
\text { family conflict }\end{array}$ & $\begin{array}{c}10 \\
(5.4)\end{array}$ & $\begin{array}{c}30 \\
(3.1)\end{array}$ & $\begin{array}{c}12 \\
(1.5)\end{array}$ & $\begin{array}{c}18 \\
(2.2)\end{array}$ & $\begin{array}{c}15 \\
(5.1)\end{array}$ & $\begin{array}{c}85 \\
(2.8)\end{array}$ \\
\hline $\begin{array}{l}\text { Other major } \\
\text { stresses }\end{array}$ & $\begin{array}{c}50 \\
(27.2)\end{array}$ & $\begin{array}{c}203 \\
(21.0)\end{array}$ & $\begin{array}{c}123 \\
(15.2)\end{array}$ & $\begin{array}{c}148 \\
(18.1)\end{array}$ & $\begin{array}{c}100 \\
(34.0)\end{array}$ & $\begin{array}{c}624 \\
(20.3)\end{array}$ \\
\hline
\end{tabular}


The majority of participants reported occasional ("minsan" or "some of the time") experience of stress. This was uniformly reported in all study areas. Participants from Metro Manila were most stressed as shown by highest prevalence of frequent ("madalas or "several periods") stress. The opposite was reported in Batangas which had the lowest rate of frequent stress. Permanent stress was infrequently felt in all study areas (Table 6).

Table 6. Frequency and percent distribution of participants by occurrence of stress within the past year

\begin{tabular}{lcccccc}
\hline $\begin{array}{c}\text { Occurrence } \\
\text { of stress }\end{array}$ & $\begin{array}{c}\text { Metro } \\
\text { Manila } \\
(\mathbf{n}=\mathbf{1 8 4})\end{array}$ & $\begin{array}{c}\text { Bulacan } \\
(\mathbf{n}=\mathbf{9 6 9 )}\end{array}$ & $\begin{array}{c}\text { Quezon } \\
(\mathbf{n}=\mathbf{8 0 9 )}\end{array}$ & $\begin{array}{c}\text { Batangas } \\
(\mathbf{n}=\mathbf{8 1 6})\end{array}$ & $\begin{array}{c}\text { Rizal } \\
(\mathbf{n}=\mathbf{2 9 4})\end{array}$ & $\begin{array}{c}\text { TOTAL } \\
(\mathbf{n}=\mathbf{3 0 7 2})\end{array}$ \\
\hline Never & 21 & 136 & 148 & 176 & 39 & 520 \\
& $(11.4)$ & $(14.0)$ & $(18.3)$ & $(21.6)$ & $(13.3)$ & $(16.9)$ \\
Some & 119 & 652 & 563 & 561 & 185 & 2080 \\
periods & $(64.7)$ & $(67.3)$ & $(69.6)$ & $(68.8)$ & $(62.9)$ & $(67.7)$ \\
Several & 39 & 146 & 73 & 60 & 54 & 372 \\
periods & $(21.2)$ & $(15.1)$ & $(9.0)$ & $(7.4)$ & $(18.4)$ & $(12.1)$ \\
Permanent & 5 & 35 & 25 & 19 & 16 & 100 \\
& $(2.7)$ & $(3.6)$ & $(3.1)$ & $(2.3)$ & $(5.4)$ & $(3.3)$ \\
\hline
\end{tabular}

A moderate amount of financial stress was felt by majority. Similar with general stress, participants from Metro Manila reported the highest proportion of high/severe financial stress. Participants from Bulacan and Rizal were least stressed as shown by having the highest proportion of little and no stress in Table 7.

Table 8 shows that occurrence of general stress within the past year was higher among females $(p<0.001)$, younger age-group $(p=0.006)$, and among college level $(p<0.001)$. Furthermore, level of current financial stress was high to severe among older age-group $(p=0.004)$, and among widow/widower/separated $(p<0.0001)$ as shown in Table 9.

Table 7. Frequency and percent distribution of participants by level of current financial stress

\begin{tabular}{lcccccc}
\hline $\begin{array}{c}\text { Level of } \\
\text { financial } \\
\text { stress }\end{array}$ & $\begin{array}{c}\text { Metro } \\
\text { Manila } \\
(\mathbf{n}=\mathbf{1 8 4})\end{array}$ & $\begin{array}{c}\text { Bulacan } \\
(\mathbf{n}=\mathbf{9 6 9 )}\end{array}$ & $\begin{array}{c}\text { Quezon } \\
(\mathbf{n}=\mathbf{8 0 9})\end{array}$ & $\begin{array}{c}\text { Batangas } \\
(\mathbf{n}=\mathbf{8 1 6})\end{array}$ & $\begin{array}{c}\text { Rizal } \\
(\mathbf{n}=\mathbf{2 9 4})\end{array}$ & $\begin{array}{c}\text { TOTAL } \\
(\mathbf{n}=\mathbf{3 0 7 2})\end{array}$ \\
\hline None & 18 & 215 & 141 & 129 & 92 & 595 \\
& $(9.8)$ & $(22.2)$ & $(17.4)$ & $(15.8)$ & $(31.3)$ & $(19.4)$ \\
Little & 22 & 153 & 126 & 111 & 22 & 434 \\
& $(12.0)$ & $(15.8)$ & $(15.6)$ & $(13.6)$ & $(7.5)$ & $(14.1)$ \\
Moderate & 111 & 495 & 470 & 500 & 142 & 1718 \\
& $(60.3)$ & $(51.1)$ & $(58.1)$ & $(61.3)$ & $(48.3)$ & $(55.9)$ \\
High/ & 33 & 106 & 72 & 76 & 38 & 325 \\
severe & $(17.9)$ & $(10.9)$ & $(8.9)$ & $(9.3)$ & $(12.9)$ & $(10.6)$ \\
\hline
\end{tabular}

\section{Discussion}

\section{Civil status}

The LIFECARE cohort had a higher proportion of married respondents $(65.7 \%)$ compared with the reported national prevalence (45.4\%) for 2010. Unlike our data which showed majority of women comprising the married subgroup, national data reported equal proportion between sexes (male $49.6 \%$, female $50.4 \%$ ). There were more live-in or common-law marriages in our cohort at $11.5 \%$ compared with the national prevalence of $5.4 \%$. In our cohort, among the never married group, a higher proportion of males than females was reported, and this is similar to the 2010 national census. ${ }^{12}$ Meanwhile, the proportion of females was higher than males among those who were widowed.

Table 8. Frequency and percent distribution of participants by occurrence of stress within the past year and demographic characteristics

\begin{tabular}{|c|c|c|c|c|c|}
\hline & \multicolumn{4}{|c|}{ Occurrence of stress } & \multirow{2}{*}{$p$-value } \\
\hline & Never & Some periods & Several periods & Permanent & \\
\hline \multicolumn{6}{|l|}{ Sex } \\
\hline Male $(n=1329)$ & $283(21.29)$ & 897 (67.49) & $113(8.50)$ & $36(2.71)$ & $<0.0001$ \\
\hline Female $(n=1743)$ & $237(13.60)$ & $1183(67.87)$ & $259(14.86)$ & $64(3.67)$ & \\
\hline \multicolumn{6}{|l|}{ Age (years) } \\
\hline $20-29(n=861)$ & $119(13.82)$ & $609(70.73)$ & 109 (12.66) & $24(2.79)$ & 0.006 \\
\hline $30-39(n=1102)$ & $177(16.06)$ & $762(69.15)$ & $124(11.25)$ & $39(3.54)$ & \\
\hline $40-50(n=1109)$ & $224(20.20)$ & $709(63.93)$ & $139(12.53)$ & $37(3.34)$ & \\
\hline \multicolumn{6}{|l|}{ Civil status } \\
\hline Single $(n=581)$ & $97(16.70)$ & $391(67.30)$ & $74(12.74)$ & $19(3.27)$ & 0.438 \\
\hline Married $(n=2018)$ & $345(17.10)$ & $1382(68.48)$ & $230(11.40)$ & $61(3.02)$ & \\
\hline Widow/Widower $(n=65)$ & $11(16.92)$ & $37(56.92)$ & $15(23.08)$ & $2(3.08)$ & \\
\hline Separated $(n=56)$ & $11(19.64)$ & $35(62.50)$ & $8(14.29)$ & $2(3.57)$ & \\
\hline Live-in $(\mathrm{n}=352)$ & $56(15.91)$ & $235(66.76)$ & $45(12.78)$ & $16(4.55)$ & \\
\hline \multicolumn{6}{|l|}{ Employment status } \\
\hline Employed $(\mathrm{n}=2068)$ & $356(17.21)$ & $1409(68.13)$ & $231(11.17)$ & $72(3.48)$ & 0.108 \\
\hline Unemployed $(\mathrm{n}=1004)$ & $164(16.33)$ & $671(66.83)$ & $141(14.04)$ & $28(2.79)$ & \\
\hline \multicolumn{6}{|l|}{ Educational attainment } \\
\hline Elementary level $(\mathrm{n}=615)$ & $150(24.39)$ & $376(61.14)$ & $63(10.24)$ & $26(4.23)$ & $<0.0001$ \\
\hline High school level $(n=1650)$ & $274(16.61)$ & 1147 (69.52) & $178(10.79)$ & $51(3.09)$ & \\
\hline College level $(n=800)$ & $93(11.63)$ & $553(69.13)$ & $131(16.38)$ & $23(2.88)$ & \\
\hline
\end{tabular}


Table 9. Frequency and percent distribution of participants by level of current financial stress and demographic characteristics

\begin{tabular}{|c|c|c|c|c|}
\hline & \multicolumn{3}{|c|}{ Level of Current Financial Stress } & \multirow[b]{2}{*}{$p$-value } \\
\hline & None/Little & Moderate & High/Severe & \\
\hline \multicolumn{5}{|l|}{ Sex } \\
\hline Male $(n=1329)$ & 465 (34.99) & $730(54.93)$ & $134(10.08)$ & 0.284 \\
\hline Female $(\mathrm{n}=1743)$ & $564(32.36)$ & $988(56.68)$ & $191(10.96)$ & \\
\hline \multicolumn{5}{|l|}{ Age (years) } \\
\hline $20-29(\mathrm{n}=861)$ & $314(36.47)$ & $474(55.05)$ & $73(8.48)$ & 0.004 \\
\hline $30-39(n=1102)$ & 369 (33.48) & $626(56.81)$ & $107(9.71)$ & \\
\hline $40-50(\mathrm{n}=1109)$ & $346(31.20)$ & $618(55.73)$ & $145(13.07)$ & \\
\hline \multicolumn{5}{|l|}{ Civil status } \\
\hline Single $(n=581)$ & $229(39.41)$ & $299(51.46)$ & $53(9.12)$ & $<0.0001$ \\
\hline Married $(n=2018)$ & $644(31.91)$ & 1159 (57.43) & 215 (10.65) & \\
\hline Widow/Widower $(\mathrm{n}=65)$ & $21(32.31)$ & $31(47.69)$ & $13(20.00)$ & \\
\hline Separated $(\mathrm{n}=56)$ & $19(33.93)$ & $24(42.86)$ & $13(23.21)$ & \\
\hline Live-in $(\mathrm{n}=352)$ & $116(32.95)$ & $205(58.24)$ & $31(8.81)$ & \\
\hline \multicolumn{5}{|l|}{ Employment status } \\
\hline Employed $(\mathrm{n}=2068)$ & $691(33.41)$ & $1163(56.24)$ & $214(10.35)$ & 0.801 \\
\hline Unemployed $(n=1004)$ & $338(33.67)$ & $555(55.28)$ & $111(11.06)$ & \\
\hline \multicolumn{5}{|l|}{ Educational attainment } \\
\hline Elementary level $(\mathrm{n}=615)$ & $194(31.54)$ & $359(58.37)$ & $62(10.08)$ & 0.372 \\
\hline High school level $(n=1650)$ & $563(34.12)$ & $921(55.82)$ & $166(10.06)$ & \\
\hline College level $(n=800)$ & $268(33.50)$ & 435 (54.37) & $97(12.13)$ & \\
\hline
\end{tabular}

\section{Employment status}

The employment rate of our cohort $(67.4 \%)$ is lower than the reported $92.4 \%$ national employment rate at the time the LIFECARE study recruitment was conducted in 2009. ${ }^{13}$ The difference may be attributed first, to the included age group and second, to the definition of status of employment. The national survey accounted for individuals aged 15 years and above while the present cohort included only individuals aged 20-50 years. Secondly, the definition of employment status was different. The national survey defined employment as having at least one hour of work during the reference period. ${ }^{14}$ The LIFECARE study defined employment as regular, not regular or self-employed as reported by the participants.

There is also a substantial disparity in the proportion of employed males and females. In spite of more women having higher educational attainment, women lagged behind in employment. Our data is consistent with national surveys in the past decade which uniformly reported a larger participation of males in the workforce. For the years 1999-2010, the national labor force participation rate for males was $79.9-84.8 \%$. Our cohort reported $86.1 \%$ of men to be employed. For women, only $53.3 \%$ of the females in our cohort were working. This is similar with the reported female labor force participation rate of $48.7-54 \%$ for 1999 $2010 .^{15}$

\section{Educational attainment}

In the present survey, almost all the participants had formal education. While not directly comparable, our data appears to be similar with the reported simple and functional literacy rate of Filipinos in 2008. The simple literacy rate (i.e., having the ability of a person to read and write with understanding of simple messages in any language or dialect) was reported to be at $95.6 \%$ which is comparable with our cohort wherein $99.8 \%$ had formal education. The more sophisticated functional literacy rate (i.e., having the ability to read, write and compute or comprehend) of Filipinos in 2008 was $86.4 \%$. Persons who graduated from high school or completed a higher level of education are considered functionally literate ${ }^{16}$. In our cohort, $79.8 \%$ achieved at least high school education. This reflects good access to education of the population in the study sites.

In our cohort, a higher proportion of females achieved at least high school level of education or are considered functionally literate ( $81.9 \%$ vs $76.9 \%$ for males). The national survey of 2008 also showed slightly higher simple (96.1\%) and functional $(88.7 \%)$ literacy rates of women than men who had simple and functional literacy rates of $94.3 \%$ and $84.2 \%$, respectively. ${ }^{16}$ These data reflect good and successful access and retention of Filipinas in school. This is evidence of the high level of gender equality in the Philippines. ${ }^{17}$ It may also imply underachievement of males in school or early transfer of males from education to participation in the labor force as a consequence of economic need as what is seen in many developing countries. ${ }^{18}$

\section{Demographics and stress}

Employment-related events were the most prevalent stressors experienced by the cohort and this was followed by health concerns. Among the study areas, Metro Manila, which is located in the National Capital Region and is the most urbanized study area, reported to have the highest occurrence of stress for both general and financial stress. The largely rural areas of Batangas and Rizal had the lowest proportions of general and financial stress, respectively. Women, younger age-group, and those who reached college 
level of education reported to have higher level of general stress. On the other hand, level of current financial stress was felt more severely by older age-group and those who did not have stable domestic status (widow/widower/separated).

There is paucity of published local epidemiological studies on stress among Filipinos. Several published studies tackle stress in relation to specific occupation such as among teachers, supervisors, nurses and nursing students. ${ }^{19-22} \mathrm{~A}$ number of studies discuss the stress profiles of migrant or overseas Filipino workers. ${ }^{23,24}$ Investigations on wide scale stress profiles of Filipinos dwelling in our homeland are lacking. Furthermore, relationship between demographic factors and stress and illness has not been extensively investigated.

\section{Conclusion}

The Philippine LIFECARE cohort is composed of participants who were mostly married and employed. Almost all had formal education and majority attained at least high school level. Majority of the cohort reported occasional experience of general stress and moderate level of financial stress. Loss of job was the most common stressor experienced in the past year. Occurrence of general stress was higher among females, younger age-group, and those who reached college level of education. High to severe current financial stress was greater among widow/widower/separated and older age-group.

While the relationship between psychosocial stress and physical illness had not been established in this study, there is a need to investigate demographics and psychosocial stress, and their implications in increasing adverse health outcomes in general, and cardiovascular risk in particular.

\section{Acknowledgments}

The following organizations provided financial assistance to the LIFECARE Philippines project: Pfizer International, Pfizer Asia, Pfizer Philippines, Department of Health (Philippines), Philippine Council for Health Research and Development, Diabetes Philippines, Philippine Society of Hypertension, and the Philippine Lipid and Atherosclerosis Society. The University of the Philippines Manila through the College of Medicine, and the National Institutes of Health's Institute of Clinical Epidemiology have also supported the project. The assistance of our support team (Rona May de Vera, Rachel Longalong, and Alma Amparo), field interviewers, and barangay health workers is gratefully acknowledged.

\section{References}

1. Department of Health National Epidemiology Center. The 2009 Philippine Health Statistics [Online]. 2009 [cited 2013 Dec]. Available from http://www.doh.gov.ph/sites/default/files/PHILIPPINE\%20HEALTH\%20STA TISTICS\%202009_0.pdf.

2. Department of Health. Leading Causes of Mortality [Online]. 2013 [cited 2013 Dec]. Available from http://www.doh.gov.ph/ node/198.html.
3. Gupta R, Deedwania PC, Sharma K, et al. Association of educational, occupational and socioeconomic status with cardiovascular risk factors in Asian Indians: a cross-sectional study. PLoS ONE. 2012;7(8):e44098.

4. $\mathrm{Yu} Z$, Nissinen A, Vartiainen E, et al. Associations between socioeconomic status and cardiovascular risk factors in an urban population in China. Bull World Health Organ. 2000; 78(11):1296-1305.

5. Bobak M, Hertzman C, Skodova Z, Marmot M. Socioeconomic status and cardiovascular risk factors in the Czech Republic. Int J Epidemiol. 1999; 28(1):46-52.

6. Luoto R, Pekkanen J, Uutela A, Tuomilehto J. Cardiovascular risks and socioeconomic status: differences between men and women in Finland. J Epidemiol Community Health. 1994; 48:348-54.

7. Thawornchaisit P, de Looze F, Reid CM, Seubsman S, Sleigh AC, and Thai Cohort Study Team. Health risk factors and the incidence of hypertension: 4year prospective findings from a national cohort of 60569 Thai Open University students. BMJ Open. 2013; 3(6): e002826.

8. Schwandt H, Coresh J, Hindin MJ. Marital status, hypertension, coronary heart disease, diabetes, and death among African American women and men: incidence and prevalence in the atherosclerosis risk in communities (ARIC) study participants. J Fam Issues. 2010; 31(9):1211-29.

9. Nabi H, Kivimaki M, Batty GD, et al. Increased risk of coronary heart disease among individuals reporting adverse impact of stress on their health: the Whitehall II prospective cohort study. Eur Heart J. 2013; 34 (34): 2697-705.

10. Sy RG, Llanes EJB, Reganit PF, et al. Socio-Demographic Factors and the Prevalence of Metabolic Syndrome Among Filipinos from the LIFECARE Cohort. J Atheroscler Thromb. 2014; 21 Suppl 1:S9-17.

11. Castillo-Carandang NT, Sison OT, Grefal ML, et al. A community-based validation study of the Short-Form 36 Version 2 Philippines (Tagalog) in two cities in the Philippines. PLoS ONE. 2013; 8(12): e83794.

12. National Statistics Office. Gender Quickstat $-4^{\text {th }}$ Quarter 2013 [Online]. $2013 \quad$ [cited $2013 \quad$ Dec]. Available from http://www.census.gov.ph/statistics/quickstat.

13. National Statistics Office. Quickstat: A Monthly Update of Philippine Statistics [Online]. 2009 [cited 2013 Dec]. Available from http://www.census.gov.ph/sites/default/files/attachments/ird/quickstat/qs0912 tb.pdf.

14. Bureau of Labor and Employment Statistics \& National Statistics Office. Primer on Philippine Labor Force Survey [Online]. 2011 [cited 2014 Feb]. Available from http://www.bles.dole.gov.ph/PUBLICATIONS/primers/ LFS_April2011.pdf.

15. International Labor Organization. Labor force participation rate (ILO estimates; by sex and age group) for Philippines [Online]. 2013 [cited 2014 Feb]. Available from http://www.ilo.org/empelm/what/ WCMS_114240/lang-en/index.htm.

16. Technical Education and Skills Development Authority. Labor Market Intelligence Report, Highlights of the 2008 Functional Literacy, Education and Mass Media Survey (FLEMMS) [Online]. 2008 [cited 2013 Dec]. Available from http://www.tesda.gov.ph/uploads/File/ LMIR2011/ST-PO\%2006-03$2011 \% 20 \% 20$ (FLEMMS).pdf.

17. World Economic Forum. The Global Gender Gap Report 2013 [Online]. 2013 [cited 2014 Feb]. Available from http://www3.weforum.org/docs/ WEF_GenderGap_Report_2013.pdf.

18. USAID. Education from a Gender Equality Perspective [Online]. May 2008 [cited 2014 Feb]. Available from http://www.ungei.org/resources/ files/Education_from_a_Gender_Equality_Perspective.pdf.

19. Roxas CC. Stress among public elementary school teachers. University of the Cordilleras. 2009; 1(4): 86-108.

20. Lu JL. Work and stress among supervisors in selected industries in the context of a globalized labour production [Online]. 2012 [cited 2014 Feb]. Available from http://www.ilo.org/public/english/iira/documents/newsletters/nov2012/ lu.pdf

21. Labrague LJ. Stress, stressors, and stress responses of student nurses in a government nursing school. Health Science Journal. 2013; 7(4): 424-35.

22. De Castro AB, Cabrera SL, Gee GC, Fujishiro K, Tagalog EA. Occupational health and safety issues among nurses in the Philippines. AAOHN J. 2009; 57(4):149-57.

23. Andal EM. A pilot study quantifying Filipino nurses' perception of stress. Calif J Health Promot. 2006; 4 (4): 88-95.

24. Huang FF, Yang HH. The effects of nationality differences and work stressors on work adjustment for foreign nurse aides. BMC Health Serv Res. 2011; 11(1):192. 